\title{
Linear Atrophoderma of Moulin: A Case Report and Review of the Literature
}

\author{
Kuanjira Wongkietkachorn Julphat Intarasupht Chutika Srisuttiyakorn \\ Kobkul Aunhachoke Artit Nakakes Nucha Niumpradit \\ Division of Dermatology, Department of Medicine, Phramongkutklao Hospital, \\ Bangkok, Thailand
}

\section{Key Words}

Atrophoderma of Moulin · Linear dermatosis · Blaschko's lines

\begin{abstract}
Linear atrophoderma of Moulin (LAM) is a rare dermatosis in childhood and early adolescence. The exact etiology of LAM is still obscure. Several treatment modalities were reported but none was consistently successful. We report a case of LAM in which a favorable outcome was obtained with topical calcipotriol. The relevant literature is also reviewed.
\end{abstract}

\section{Introduction}

Linear atrophoderma of Moulin (LAM) is a rare linear dermatosis. The disease usually presents in childhood or early adolescence as asymptomatic hyperpigmented atrophic linear bands along Blaschko's lines without prior inflammation or sclerotic appearance. LAM usually progresses as a linear atrophic lesion in the first few months; then the lesion ceases to progress and persists. We report a case of LAM with partial response to topical calcipotriol.

\section{Case Presentation}

A 15-year-old male Thai presented for a 1-month history of two progressive asymptomatic depressed linear plaques along Blaschko's lines (fig. 1). The first lesion was on the trunk, extending laterally from the umbilicus toward the middle of the right lower back at 
the level of T10 of the thoracic spine. The second lesion was on his right leg, extending from the middle of the popliteal fossa toward the medial malleolus. There was no sign of inflammation or sclerosis on the lesion. The remaining results from the physical examination were unremarkable. There was no underlying disease, and no one in his family had the same skin lesion.

Histopathology of the lesion revealed epidermal atrophy without any change in the dermis or collagen bundle. The elastic stain showed normal elastic tissue.

Treatment options were discussed and explained to the patient. He agreed to try topical calcipotriol twice a day on the lesion on his abdominal wall, leaving the lesion on his back as a control. After 3 months of follow-up, clinical improvement was observed in the treated area in comparison to the untreated control area, which was appreciated by the patient (fig. 2). After successful treatment of the abdominal area, we asked the patient to apply topical calcipotriol to all lesions; unfortunately, there was no additional improvement of the abdominal and leg lesions after further 2 months of treatment. Based on this result, we consider topical calcipotriol treatment an effective approach in the early stage of LAM.

\section{Discussion}

LAM was first described by Moulin in 1992 as an acquired unilateral hyperpigmented atrophic band along Blaschko's lines [1]. The classic histopathology revealed no inflammation or sclerotic change. LAM usually progresses as a linear atrophic lesion in the first few months; then the lesion ceases to progress and persists. The lesion of LAM usually resists most treatment options; however, the prognosis is excellent because there is no systemic condition associated with the disease.

The etiology of LAM remains unclear. A study of the atrophic component of LAM by ultrasonography revealed that subcutaneous volume reduction was the cause of the atrophic appearance, not dermal atrophy [2].

Even though the clinical manifestation of LAM is rather unique, histopathology of LAM is quite inconspicuous. Hematoxylin and eosin staining usually shows hyperpigmentation only in basal epidermal layers without abnormal collagen or elastic fibers in the dermis or any obvious inflammation $[1,3,4]$. There may be some perivascular lymphocytic infiltration $[2$, 5-12], acanthosis [7, 8], epidermal atrophy [6], altered collagen in the dermis [2, 5-7, 9, 10, 13], and decreased or fragmented elastic tissue [14]. Once there is some alteration of the collagen component, the diagnosis should be atrophoderma of Pasini and Pierini (APP) [15].

The differential diagnosis of LAM includes APP, linear scleroderma, lichen striatus, and focal dermal hypoplasia. Linear scleroderma and APP may show a segmental atrophic plaque similar to LAM. Abnormal collagen fibers in the histopathological findings and dermal tissue reduction in the ultrasound imaging are helpful for the diagnosis of linear scleroderma and APP, respectively $[2,14]$. Considering the clinical manifestation of LAM, i.e. the characteristic atrophic band and hyperpigmentation, the following conditions may resemble LAM: incontinentia pigmenti, nevus of Ito, linear and whorled nevoid hypermelanosis, and linear lichen planus.

In general, LAM is typically a self-limited disease. Progression of the lesions usually stops within a few months without any internal organ involvement. There have also been cases of LAM in association with idiopathic urticaria and hypothyroidism [8]. There are reports of variants such as multiple telangiectatic macules accompanying the atrophic plaque [11], bilateral LAM $[8,11]$, and LAM preceded by inflammation [8]. 
Currently, there is no standard treatment regimen for LAM. However, partial improvement was obtained with intravenous penicillin together with topical PUVA therapy, oral Potaba (potassium para-aminobenzoate), high-dose vitamin E (400 IU/day), and topical clobetasol propionate $[7,11,12]$. Other cases were treated with topical corticosteroids, topical corticosteroids and heparin, high-dose penicillin, and photo protection - all without improvement $[5,6,9,10,13]$.

In our case, the patient was treated with topical calcipotriol twice a day. Calcipotriol is a synthetic 1,25-dihydroxyvitamin D3, which has efficacy in the treatment of plaque-type morphea. After 3 months, the progression of the lesion stopped, and partial improvement of the treated area occurred. Topical calcipotriol was applied to both the legs and abdominal area for the next 2 months, but interestingly, no further clinical improvement was observed. This finding suggests that early treatment is of great importance.

In conclusion, we reported a case of classic LAM with partial response to topical calcipotriol treatment. We suggest that treatment should be started early in order to achieve good response.

\section{References}

1 Moulin G, Hill MP, Guillaud V, Barrut D, Chevallier J, Thomas L: Acquired atrophic pigmented band-like lesions following Blaschko's lines (in French). Ann Dermatol Venereol 1992;119:729-736.

-2 Norisugi O, Makino T, Hara H, Matsui K, Furuichi M, Shimizu T: Evaluation of skin atrophy associated with linear atrophoderma of Moulin by ultrasound imaging. J Am Acad Dermatol 2011;65:232-233.

-3 Cecchi R, Giomi A: Linear atrophoderma of Moulin. Acta Derm Venereol 1997;77:485.

-4 López N, Gallardo MA, Mendiola M, Bosch R, Herrera E: A case of linear atrophoderma of Moulin (in Spanish). Actas Dermosifiliogr 2008;99:165-167.

5 Baumann L, Happle R, Plewig G, Schirren CG: Atrophodermia linearis Moulin. A new disease picture, following the Blaschko lines (in German). Hautarzt 1994;45:231-236.

-6 Wollenberg A, Baumann L, Plewig G: Linear atrophoderma of Moulin: a disease which follows Blaschko's lines. Br J Dermatol 1996;135:277-279.

7 Artola Igarza JL, Sánchez Conejo-Mir J, Corbí Llopis MR, Linares Barrios M, Casals Andreu M, Navarrete Ortega M: Linear atrophoderma of Moulin: treatment with Potaba. Dermatology 1996;193:345-347.

8 Browne C, Fisher BK: Atrophoderma of moulin with preceding inflammation. Int J Dermatol 2000;39: 850-852.

9 Rompel R, Mischke AL, Langner C, Happle R: Linear atrophoderma of Moulin. Eur J Dermatol 2000;10: 611-613.

-10 Martin L, Georgescu V, Nizard S, Happle R, Estève E: Unilateral atrophoderma following Blaschko's lines: Blaschkolinear morphoea or Moulin's linear atrophoderma? (in French). Ann Dermatol Venereol 2002;129:431-432.

11 Utikal J, Keil D, Klemke CD, Bayerl C, Goerdt S: Predominant telangiectatic erythema in linear atrophoderma of Moulin: novel variant or separate entity? Dermatology 2003;207:310-315.

12 Zampetti A, Antuzzi D, Caldarola G, Celleno L, Amerio P, Feliciani C: Linear atrophoderma of Moulin. Eur J Dermatol 2008;18:79-80.

13 Larregue M, Vabres P, Rat JP, Auriol F, de Giacomoni P: Atrophodermie pigmentée linéaire de Moulin. Ann Dermatol Venereol 1995;122:73s-74s.

14 Ang G, Hyde PM, Lee JB: Unilateral congenital linear atrophoderma of the leg. Pediatr Dermatol 2005;22:350-354.

15 Ang GC, Lee JB: Linear atrophoderma of Moulin: is it a single disease? J Am Acad Dermatol 2005;52: 923-924. 
Case Reports in
Dermatology

Case Rep Dermatol 2013;5:11-14

DOI: $\underline{10.1159 / 000346747}$

Wongkietkachorn et al.: Linear Atrophoderma of Moulin: A Case Report and Review of the Literature

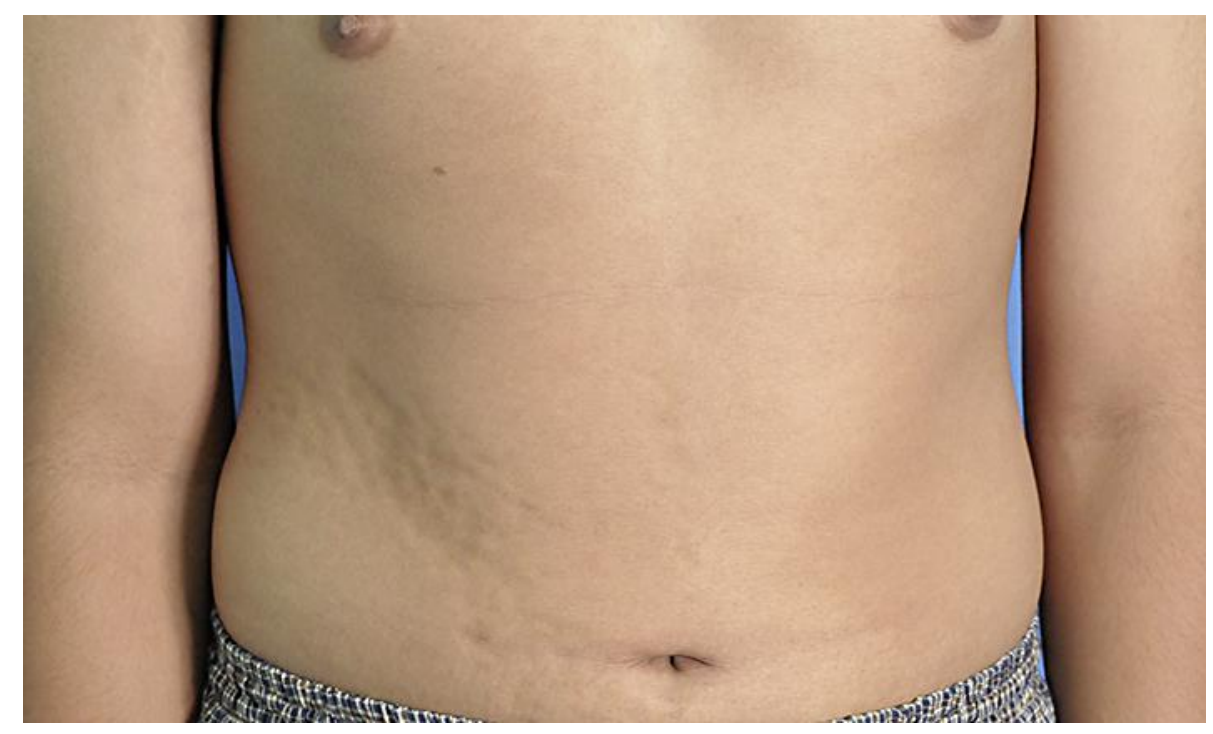

Fig. 1. Initial clinical presentation.

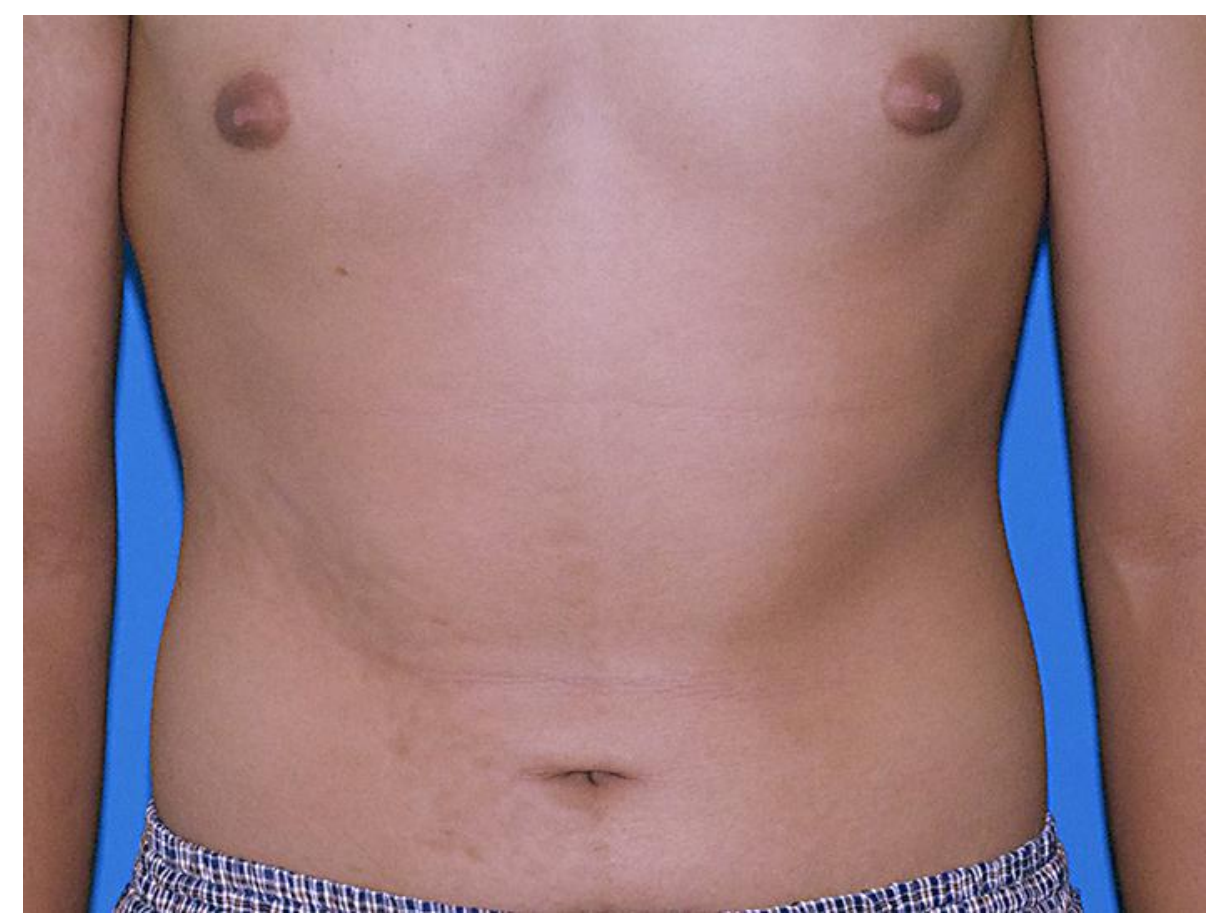

Fig. 2. Clinical presentation 3 months after topical calcipotriol treatment. 